\title{
A Comparison between New Iterative Solutions of Non-linear Oscillator Equation
}

\author{
M. Khalid \\ Department of Mathematical Sciences \\ Federal Urdu University of Arts, Sciences \& Techonology \\ University Road, Karachi-75300, Pakistan \\ Uroosa Arshad \\ Department of Mathematical Sciences \\ Federal Urdu University of Arts, Sciences \& Technology \\ University Road, Karachi-75300, Pakistan
}

\author{
Mariam Sultana \\ Department of Mathematical Sciences \\ Federal Urdu University of Arts, Sciences \& Technology \\ University Road, Karachi-75300, Pakistan \\ Muhammad Shoaib \\ Department of Physics \\ Federal Urdu University of Arts, Sciences \& Technology \\ University Road, Karachi-75300, Pakistan
}

\begin{abstract}
The prime focus is on a Van der Pol-Duffing oscillator in this paper. A newly proposed method, namely; the Perturbation Iteration Algorithm (PIA) and an Alternative Variation Iteration Method (AVIM) is used to solve governing equations. The study outlines the significant features of the two methods. The beauty of the paper lies in the error analysis between exact solutions and approximate solutions obtained by these two methods which proves that approximate solutions obtained by Alternative Variation Iteration Method converge very rapidly to the exact solutions. Both methods provide analytical solution in the form of a convergent series with components that are easily computable, requiring no linearization or small perturbation
\end{abstract}

\section{Keywords}

Van der Pol-Duffing oscillator, Perturbation Iteration Algorithm, Alternative Variational Iteration Method, Convergent Series, Efficient, Convergence.

\section{INTRODUCTION}

In the past few decades, chaos and chaotic systems have received a flurry of research effort. Such systems are nonlinear by nature and can be found in many natural and man made systems. These [1] are recognized by their great sensitivity to initial conditions. The evaluation of nonlinear vibrations and oscillations is essential in every engineering science. These oscillator equations provide an important mathematical model for dynamical systems having a single unstable fixed point, along with a single stable limit cycle. All natural and engineering sciences [2, 3], along with many physical problems [4 5] possess examples of phenomena like these. Up until now, various aspects of this problem have been analyzed by different authors; areas such as vibration amplitude control, synchronization dynamics, and additive resonances [6]-9]. Its uses include modeling optical disability in a dispersive medium, the refractive index in which depends on the optical intensity [10]. Other applications may have certain flow induced structural vibration problems [11]. Understanding of dynamical behaviors becomes practical when dealing with nonlinear oscillations. There is a whole world of literature on this topic and cannot possibly be described here in detail. Some of the more recent works regarding the Van der Pol-Duffing oscillator by eminent researchers, however, have been mentioned in the open literature [11-17]

Perturbation Iteration Algorithm are classical ones that have been around for over a century to find approximate analytical solutions. Several different Perturbation techniques and their variants have developed; including the method of multiple scales, the renormalization method, and the method of averaging, the Lindstedt-Poincare method, and the method of matched asymptotic expansions [18, 19]. One major drawback of the Perturbation Iteration Algorithm is the necessity of a small parameter; the small parameter may also have to be artificially introduced into the equations. The solutions, ergo, have a limited range of validity. The solutions, although being valid for weak nonlinear problems, are not admissible for strong nonlinear ones. Several new techniques have been proposed to obtain admissible solutions that exclude the small parameter assumption. Recently, a class of alternative Perturbation Iteration Algorithms has been put forward. The basics of the algorithms were drafted for the first-order differential equations by Pakdemirli et al. [20]. Several iteration algorithms can be derived by taking different number of terms in the perturbation expansions and adopting different order of correction terms in the Taylor Series expansions. The Perturbation Iteration Algorithm is called $P I A(n, m)$ where $n$ represents the correction terms in the perturbation expansion and $m$ the highest order derivative term in the Taylor Series. One main plus is no requirement of initial assumptions or transformation of the equations to another form.

Variational Iteration Method has been widely used to handle linear and nonlinear models. The main property of the method is its flexibility and ability to solve nonlinear equations accurately and conveniently. Some modifications for improving the convergence speed and lengthening the interval of convergence for VIM series solutions were suggested in [21-24]. Such as, Abassy et al. [21] proposed a modification of the VIM, which was used to 
obtain approximate solutions for some nonlinear problems. Their proposed modification of the VIM reduces the computational workload and improves the speed of convergence in an effective manner [22]. Geng et al. proposed the piecewise VIM for solving Riccati differential equations [23]. The solutions obtained using the piecewise VIM provided good approximations for a larger interval, rather than in the local vicinity of the initial position. Ghorbani and Saberi-Nadjafi modified the VIM by constructing an initial trial function without unknown parameters [24]. Odibat [25] introduce an alternative approach of VIM to handle nonlinear problems.

This paper outlines a reliable comparison between two powerful methods that were recently developed. In this study, Perturbation Iteration Algorithm and the Alternative Variational Iteration Method are applied to obtain the approximate solution of some nonlinear Duffing differential equations. In the present study, we aim to extend the application of efficiency and accuracy of the methods under discussion is shown by two examples, one of them is the quadratic Duffing equation. The organization of the paper is as follows. Begin by properly introducing the Perturbation Iteration Algorithm and Alternative Variational Iteration Method in section 2. In section 3, both methods are applied to the Duffing differential equation. Two examples will be presented to show the efficiency and ease with which the proposed methods may be applied. A comparision and conclusion follows in Section 4.

\section{THE METHODS}

We will draw attention to briefly the main points of each of the two methods as follows, where details can be found in [20-25]

\subsection{Perturbation Iteration Algorithm}

In this section, a Perturbation Iteration Algorithm (PIA) proposed by Aksoy et al [20] is discussed. Authors in [20] introduced one correction term in the perturbation expansion, correction terms for the first derivatives only in the Taylor Series expansion, i.e. $n=1$, $m=1$. The algorithm is named $P I A(1,1)$. Consider a general second order differential equation,

$$
F\left(u^{\prime \prime}, u^{\prime}, u, \epsilon\right)=0
$$

With $u=u(t)$ and $\epsilon$ as the perturbation parameter. Only one correction term is taken in the perturbation expansion.

$$
u_{n+1}=u_{n}+\epsilon\left(u_{c}\right)_{n}
$$

Upon substitution of Eq.(2) into Eq.(1) and expanding in a Taylor Series with first derivatives, the following expression has been obtained.

$$
\begin{aligned}
& F\left(u_{n}^{\prime \prime}, u_{n}^{\prime}, u_{n}, 0\right)+F_{u}\left(u_{n}^{\prime \prime}, u_{n}^{\prime}, u_{n}, 0\right) \epsilon\left(u_{c}\right)_{n}+ \\
& F_{u^{\prime}}\left(u_{n}^{\prime \prime}, u_{n}^{\prime}, u_{n}, 0\right) \epsilon\left(u_{c}^{\prime}\right)_{n}+F_{u^{\prime \prime}}\left(u_{n}^{\prime \prime}, u_{n}^{\prime}, u_{n}, 0\right) \epsilon\left(u_{c}^{\prime \prime}\right)_{n}+ \\
& F_{\epsilon}\left(u_{n}^{\prime \prime}, u_{n}^{\prime}, u_{n}, 0\right) \epsilon=0
\end{aligned}
$$

where $F_{u}=\frac{\partial F}{\partial u}, F_{u^{\prime}}=\frac{\partial F}{\partial u^{\prime}}, F_{u^{\prime \prime}}=\frac{\partial F}{\partial u^{\prime \prime}}, F_{\epsilon}=\frac{\partial F}{\partial \epsilon}$. Reorganizing the equation, one can easily get

$$
\left(u_{c}^{\prime \prime}\right)_{n}+\frac{F_{u^{\prime}}}{F_{u^{\prime \prime}}}\left(u_{c}^{\prime}\right)_{n}+\frac{F_{u}}{F_{u^{\prime \prime}}}\left(u_{c}\right)_{n}=-\frac{F_{\epsilon}+\frac{F}{\epsilon}}{F_{u^{\prime \prime}}}
$$

Keeping in mind that all derivatives are evaluated at $\epsilon=0$, it is readily observed that the above equation is a variable coefficient linear second-order differential equation. Starting with an initial guess $u_{\circ}$, first $\left(u_{c}\right)_{\circ}$ is calculated from Eq. (4) and then substituted into Eq.(1) to calculate $u_{1}$. The iteration procedure is repeated using Eq.(4) and Eq.(2) until a result we may call satisfactory is obtained.

Note that for a more general algorithm, $n$ correction terms instead of one can be taken in expansion (2) which would then be an $P I A(n, m)$. The algorithm can also be generalized to a differential equation system having an arbitrary order of derivatives. In this study, we only take into account one case $n=m=1$ for the sake of simplicity, so as to keep from involvement of more Algebra in constructing iteration solutions for $P I A(1,2)$ and $P I A(1,3)$ compared to $P I A(1,1)$.

\subsection{Alternative Variational Iteration Method}

Odibat [25] proposed an alternative approach of VIM. Consider a equation in the form

$$
L u(t)+N u(t)=g(t) ; \quad t>0
$$

where the linear operator $L$ is defined as $L=\frac{d^{m}}{d t^{m}}, n \in N, N$ is a nonlinear operator and $g(t)$ is known analytic function, subjects to the initial function, subject to initial conditions,

$$
u^{(k)}(0)=c_{k} ; \quad k=0,1,2, \ldots, m-1
$$

where $c_{k}$ are real numbers. According to VIM, one can construct the correcting functional for Eq. (5) as,

$$
u_{k+1}(t)=u_{k}(t)+\int_{0}^{t}\left[\lambda(\tau)\left(L u_{k}(\tau)-N \widetilde{u}_{k}(\tau)-g(\tau)\right)\right] d \tau
$$

where $\lambda(\tau)$ is a general Lagrange multiplier, which can be identified optimally via variational theory. In general, the following Lagrange multipliers, has been received.

$$
\lambda=\frac{(-1)^{m}}{(m-1) !}(\tau-t)^{m-1} ; \quad m \geq 1
$$

Now, defines the operator $A[u]$ as,

$$
A[u]=\int_{0}^{t}\left[\lambda(\tau)\left(L u_{k}(\tau)-N \widetilde{u}_{k}(\tau)-g(\tau)\right)\right] d \tau
$$

Therefore, substituting Eq.97 into Eq.(7), the following iteration formula will be formed.

$$
u_{k+1}(t)=u_{k}(t)+A[u]
$$

and define the components $v_{k}, k=0,1,2, \ldots$ as

$$
\begin{aligned}
& v_{0}=u_{\circ} \\
& v_{1}=A\left[v_{\circ}\right] \\
& v_{2}=A\left[v_{\circ}+v_{1}\right] \\
& v_{3}=A\left[v_{\circ}+v_{1}+v_{2}\right]
\end{aligned}
$$

$$
v_{k+1}=A\left[v_{\circ}+v_{1}+v_{2}+\ldots+v_{k}\right]
$$

then consequently, $u(t)=\lim _{k \rightarrow \infty} u_{k}(t)=\sum_{k=0}^{\infty} v_{k}$. Therefore as a result, the solution of problem (5) can be derived using Eq. (10) and 
Eq.[11], in the series form,

$$
u(t)=\sum_{k=0}^{\infty} v_{k}(t)
$$

\section{NUMERICAL EXAMPLES}

In order to show the effectiveness of these two algorithms for solving the Duffing equation, some numerical examples has been presented.

\subsection{Example}

Consider the following nonlinear oscillator differential equation

$$
\frac{d^{2} u}{d x^{2}}-u+u^{2}+\left(\frac{d u}{d x}\right)^{2}-1=0
$$

with initial conditions, $u(0)=2, u^{\prime}(0)=0$. The exact solution of the above problem is $u(x)=1+\cos x$. By applying the PIA, Eq. 13 reduce to

$$
\ddot{u}^{c}=\frac{-\ddot{u}+1}{\epsilon}+u-u^{2}-\dot{u}^{2}
$$

An initial guess satisfying the initial condition should be selected So, selecting $u_{\circ}=2-x^{2}$. Using the algorithm of PIA with Eq. (14) and initial guess, the approximate solution at each step are:

$$
\begin{gathered}
u_{11}=2-\frac{1}{2} x^{2}-\frac{1}{12} x^{4}-\frac{1}{30} x^{6} \\
u_{12}=2-\frac{1}{2} x^{2}+\frac{1}{24} x^{4}-\frac{1}{45} x^{6}-\frac{89}{10080} x^{8}-\frac{5}{2592} x^{10}- \\
\frac{41}{118800} x^{12}-\frac{1}{163800} x^{14} \\
u_{13}=2-\frac{1}{2} x^{2}+\frac{1}{24} x^{4}-\frac{1}{720} x^{6}-\frac{67}{20160} x^{8}-\frac{1901}{1814400} x^{10}- \\
\frac{241}{935550} x^{12}-\frac{255553}{2179457280} x^{14}-\frac{51665461}{1307674368000} x^{16}- \\
\frac{2892761}{224550144000} x^{18}-\frac{61778699}{22817583360000} x^{20}- \\
\frac{11765053}{29875329792000} x^{22}-\frac{590337809}{14887872679680000} x^{24}- \\
\frac{1092437}{834809976000000} x^{26}-\frac{1457}{95623688160000} x^{28}- \\
\frac{1}{23342482800000} x^{30}
\end{gathered}
$$

and so on. Using the algorithm of AVIM with Eq. 13 and initial guess $u_{\circ}=2-x^{2}$, the approximate solution at each step are

$$
\begin{gathered}
v_{10}=2-\frac{1}{2} x^{2}-\frac{1}{12} x^{4}-\frac{1}{30} x^{6} \\
v_{11}=2-\frac{1}{2} x^{2}+\frac{1}{24} x^{4}-\frac{1}{45} x^{6}-\frac{89}{10080} x^{8}-\frac{5}{2592} x^{10}- \\
\frac{41}{118800} x^{12}-\frac{1}{163800} x^{14}
\end{gathered}
$$

Table 1. Average relative error of Example 1 comparision with $P I A(1,1)$ and $A V I M$

\begin{tabular}{cccc}
\hline$x$ & Exact & PIA $u_{13}$ & AVIM $v_{12}$ \\
\hline 0.0 & 2.000000000 & 2.000000000 & 2.000000000 \\
0.1 & 1.995004165 & 1.995004165 & 1.995004165 \\
0.2 & 1.980066578 & 1.980066569 & 1.980066569 \\
0.3 & 1.955336489 & 1.955336263 & 1.955336263 \\
0.4 & 1.921060994 & 1.921058685 & 1.921058685 \\
0.5 & 1.877582562 & 1.877568389 & 1.877568389 \\
0.6 & 1.825335615 & 1.825272379 & 1.825272379 \\
0.7 & 1.764842187 & 1.764615066 & 1.764615066 \\
0.8 & 1.696706709 & 1.696008265 & 1.696008265 \\
0.9 & 1.621609968 & 1.619694242 & 1.619694242 \\
1.0 & 1.540302306 & 1.535476244 & 1.535476244 \\
\hline Average & & & \\
Relative & & 0.000445311 & 0.000445311 \\
Error & & & \\
\hline
\end{tabular}

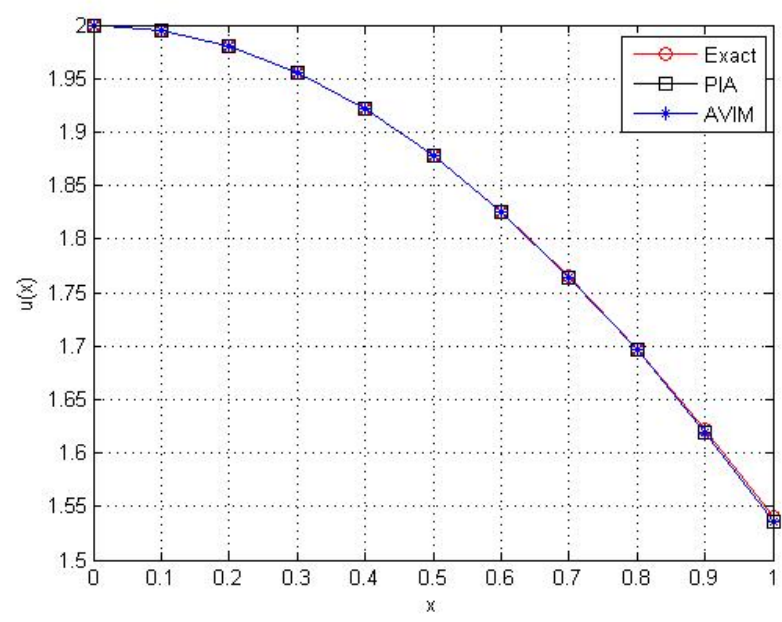

Fig. 1. Comparison of Exact and Numerical solution of PIA and $A V I M$ of Example 1

$$
\begin{aligned}
v_{12}= & 2-\frac{1}{2} x^{2}+\frac{1}{24} x^{4}-\frac{1}{720} x^{6}-\frac{67}{20160} x^{8}-\frac{1901}{1814400} x^{10}- \\
& \frac{241}{935550} x^{12}-\frac{255553}{2179457280} x^{14}-\frac{51665461}{1307674368000} x^{16}- \\
& \frac{2892761}{224550144000} x^{18}-\frac{61778699}{22817583360000} x^{20}- \\
& \frac{11765053}{29875329792000} x^{22}-\frac{590337809}{14887872679680000} x^{24}- \\
& \frac{1092437}{834809976000000} x^{26}-\frac{1457}{95623688160000} x^{28}- \\
& \frac{1}{23342482800000} x^{30}
\end{aligned}
$$

and so on. 
Table 2. Average relative error of Example 2 comparison with $P I A(1,1)$ and $A V I M$

\begin{tabular}{cccc}
\hline$x$ & Exact & $P I A u_{12}$ & $A V I M v_{11}$ \\
\hline 0.1 & 0.099833416647 & 0.092743898932 & 0.099833503766 \\
0.2 & 0.198669330795 & 0.185346581859 & 0.198672306412 \\
0.3 & 0.295520206661 & 0.277631575109 & 0.295544761414 \\
0.4 & 0.389418342309 & 0.369359701789 & 0.389532415382 \\
0.5 & 0.479425538604 & 0.460217050481 & 0.479813376489 \\
0.6 & 0.564642473395 & 0.549825131026 & 0.565724718495 \\
0.7 & 0.644217687238 & 0.637777849823 & 0.646848217682 \\
0.8 & 0.717356090900 & 0.723705739802 & 0.723119718202 \\
0.9 & 0.783326909627 & 0.807361162309 & 0.794955142884 \\
1.0 & 0.841470984808 & 0.888709214275 & 0.863375606677 \\
\hline Average & & & \\
Relative & & 0.042209072029 & 0.005611138306 \\
Error & & & \\
\hline
\end{tabular}

\subsection{Example}

Consider the following nonlinear oscillator differential equation

$$
\frac{d^{2} u}{d x^{2}}+\frac{d u}{d x}+u+u^{2} \frac{d u}{d x}=2 \cos x-\cos ^{3} x
$$

with initial conditions, $u(0)=0, u^{\prime}(0)=1$, which has the following analytical solution $u(x)=\sin x$. By applying the $P I A$ reduce Eq. [15] into

$$
\ddot{u}^{c}+u^{c}=\frac{-\ddot{u}-u}{\epsilon}-\dot{u}\left(u^{2}+1\right)+\cos x\left(2-\cos ^{2} x\right)
$$

which is the determining iteration equation for the perturbation correction term. Assuming an initial solution, successive iteration can be determined. An initial trial function $u_{10}=x$, which satisfies the initial condition is selected. Substituting this trial function into Eq. 16, solving for the correction term. The following successive iterations has been obtained

$$
\begin{gathered}
u_{10}=x \\
u_{11}=1-x^{2}-\frac{33}{32} \cos x+\frac{1}{32} \cos 3 x+\sin x+\frac{5}{8} x \sin x
\end{gathered}
$$

and so on. Using the algorithm of AVIM with Eq. (15) and initial guess $u_{\circ}=x$, the approximate solution at each step are

$$
v_{10}=x-\frac{4}{3} \cos x+\frac{1}{9} \cos x^{3}-\frac{1}{2} x^{2}-\frac{1}{6} x^{3}-\frac{1}{12} x^{4}+\frac{11}{9}
$$

and so on.

\section{DISCUSSION OF RESULTS AND CONCLUSION}

The main goal of this work is to conduct a comparative study between Alternative Variational Iteration Method and the Perturbation Iteration Algorithm. The two methods are powerful and efficient methods that both give approximations of higher accuracy and closed form solutions if existing but AVIM converges fastly as compared to PIA. Two examples of nonlinear Duffing differential equation in the form of relevant literature are presented to prove its effectiveness and efficiency. All these calculation can easily be performed through software Mathematica 9.0.

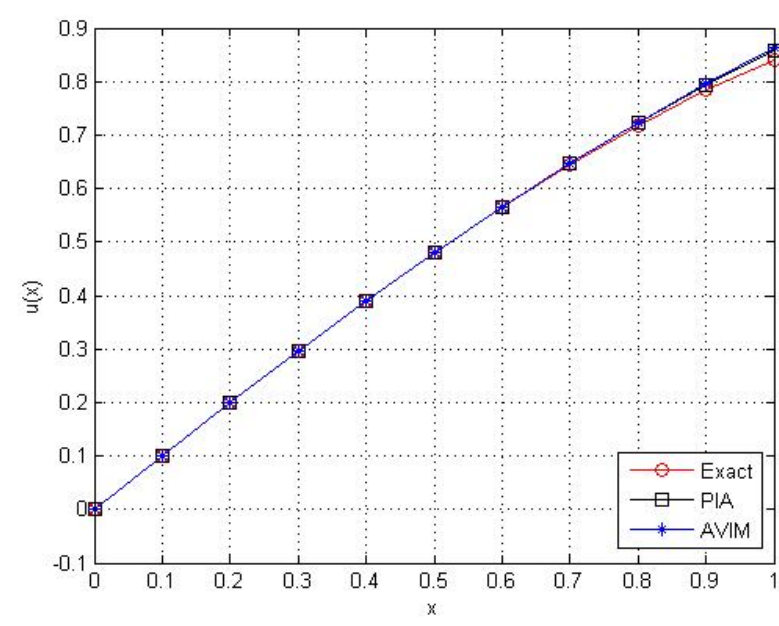

Fig. 2. Comparison of Exact and Numerical solution of PIA and AVIM of Example 2

\section{Conflict of Interests}

The authors declare that there is no conflict of interests regarding the publication of this paper.

\section{Acknowledgment}

We thank the reviewers for their thorough efforts in editing our paper and highly appreciate the comments and constructive criticism that significantly contributed in improving the quality of the publication. The authors also thank Ms. Wishaal Khalid for proofreading our research paper.

\section{REFERENCES}

[1] Kolipanos, C.H.L., Kyprianidis, I.M., Stouboulos, I.N., Anagnostopoulos, A.N. and Magafas, L. (2003) Chaotic behaviour of a fourth-order autonomous electric circuit, Chaos Solitons Fractals, vol.16, pp. 173-182.

[2] Mickens, R.E. (1996) Oscillations in Planar Dynamics Systems, World Scientific,Singapore.

[3] Mickens, R.E., Gumel, A.B. (2002) Numerical study of a non-standard finite difference scheme for the Van der Pol equation, J. Sound Vib., vol.250, pp. 955-963.

[4] Hale, J. (1969) Ordinary Differential Equations, Wiley, New York.

[5] Guckenheimer, J., Holmes, P. (1983) Nonlinear Oscillations, Dynamical Systems, and Bifurcations of Vector Fields, Springer-Verlag, New York.

[6] Maccari, A. (2008) Vibration amplitude control for a Van der Pol-Duffing oscillator with time delay, J. Sound Vib., vol.317, pp. 20-29.

[7] Njah, A.N., Vincent, U.E. (2008) Chaos synchronization between single and double wells Duffing-Van der Pol oscillators using active control, Chaos Solitons Fractals, vol.37, pp. 1356-1361.

[8] Yamapi, R., Filatrella, G. (2008) Strange attractors and synchronization dynamics of coupled Van der Pol-Duffing oscillators, Commun. Nonlinear Sci. Numer. Simul, vol.13, pp. 1121-1130. 
[9] Ji, J., Zhang, N. (2008) Additive resonances of a controlled Van der Pol-Duffing oscillator, J. Sound Vib., vol.315, pp. 22-33.

[10] Kao, Y.H., Wang, C.S. Analog study of bifurcation structures in a Van der Pol oscillator with a nonlinear restoring force, Phys. Rev. E, vol.48, pp. 2514-2520.

[11] Parlitz, U., Lauterborn, W. (1987) Period-doubling cascades and Peril's staircases of the driven Van der-Pol oscillator, Phys. Rev. A, vol.36, pp. 1428-1434.

[12] Rafei, M., Ganji, D.D., Daniali, H., Pashaei, H. (2007) The variational iteration method for nonlinear oscillators white discontinuities, J. Sound Vib., vol.305, pp. 614-620.

[13] Momani, S., Ertürk, V.S. (2008) Solutions of non-linear oscillators by the modified differential transform method, Comput. Math. Appl., vol.55, pp. 833-842.

[14] Özis, T., Yıldırım, A. (2009) Generating the periodic solutions for forcing Van der-Pol oscillators by the iteration perturbation method, Nonlinear Anal. RWA, vol.10, pp. 1984-1989.

[15] Kimiaeifar, A., Saidi, A.R., Sohouli, A.R., Ganji, D.D. (2010) Analysis of modified Van der-Pol's oscillator using He's parameter-expanding methods, Curr. Appl. Phys., vol.10, pp. 279-283.

[16] Barari, A., Omidvar, M., Ghotbi, A.R., Ganji, D.D. (2008) Application of homotopy perturbation method and variational iteration method to nonlinear oscillator differential equations, Acta Appl. Math., vol.104, pp. 161-171.

[17] Khan, Y., Madani, M., Yildirim, A., Abdou, M.A., Faraz, N. (2011) A new approach to Van der-Pol's oscillator problem, Z. Naturforsch., vol.66a, pp. 620-624.

[18] Nayfeh, A.H. (1973) Perturbation Methods, WileyInterscience, New York.

[19] Skorokhod, A.V., Hoppensteadt, F.C., Salehi, H. (2002) Random Perturbation Methods with Applications in Science and Engineering, Springer, New York.

[20] Pakdemirli, M., Aksoy, Y., H. Boyaci, H. (2011) A new perturbation-iteration approach for first order differential equations, Mathematical and Computational Applications, vol. 16, no. 4, pp. 890-899.

[21] Abassy, T.A., El-Tawil, M.A., El-Zoheiyr, H. (2007) Toward a modified variational iteration method, J. Comput. Appl. Math., vol.207, no.1, pp. 137-147.

[22] Abassy, T.A., El-Tawil, M.A., El-Zoheiyr, H. (2007) Solving nonlinear partial differential equations using the modified variational iteration Pad'e technique, J. Comput. Appl. Math., vol.207, no.1, pp. 73-91.

[23] Geng, F., Lin, Y., Cui, M. (2009) A piecewise variational iteration method for Riccati differential equations, Comput. Math. Appl., vol.58, no.11-12, pp. 2518-2522.

[24] Ghorbani, A., Saberi-Nadjafi, J. (2009) An effective modification of He's variational iteration method, Nonlinear Anal. Real World Appl., vol.10, no.5, pp. 2828-2833.

[25] Odibat, Z.M. (2010) A study on the convergence of variational iteration method, Math. Comput. Model., vol.51, pp. 1181-1192. 